\title{
Auch dauerhaft urban? Empirische Untersuchung kurz- und langfristiger Wohnumfeldwünsche junger Erwachsener als Beitrag zur Reurbanisierungsdebatte
}

\author{
Erik Seyfarth, Frank Osterhage $\mathbb{1}$, Joachim Scheiner (1) \\ Eingegangen: 23. Februar 2021 - Angenommen: 1. Juni 2021 - Online veröffentlicht: 30. August 2021
}

\begin{abstract}
Zusammenfassung
Die Wanderungen und Wohnbedürfnisse junger Erwachsener spielen im Kontext der Reurbanisierungsdebatte eine bedeutende Rolle. Regelmäßig werden sie als eine Hauptträgergruppe der Reurbanisierung identifiziert, wobei Studierende für viele Städte von besonderer Bedeutung sind. Die Befunde beziehen sich allerdings im Wesentlichen auf die Generation Y („Millenials“, geboren etwa 1980 bis 2000). Über die nachfolgende Generation Z („Post-Millennials“) ist weniger bekannt. Eine Möglichkeit zum Verständnis der Dynamik und Richtung sich möglicherweise verändernder Wohnumfeldwünsche besteht darin, junge Erwachsene danach zu fragen, wie sie nach Beendigung der Ausbildung oder später wohnen möchten. Dazu wurden Studierende der Raumplanung an der TU Dortmund befragt. Zum Verständnis der Einflussfaktoren auf die Wohnumfeldwünsche wird ein multinomiales Logitmodell geschätzt. Als zentrale Ergebnisse zeigt sich erstens, dass nur sehr wenige Studierende langfristig urban wohnen möchten,
\end{abstract}

Erik Seyfarth, Fakultät Raumplanung, Technische Universität Dortmund, August-Schmidt-Straße 10, 44221 Dortmund, Deutschland erik.seyfarth@tu-dortmund.de

Frank Osterhage, ILS - Institut für Landes- und Stadtentwicklungsforschung, Brüderweg 22-24, 44135 Dortmund, Deutschland frank.osterhage@ils-forschung.de

Prof. Dr. Joachim Scheiner, Fakultät Raumplanung, Technische Universität Dortmund, August-Schmidt-Straße 10, 44221 Dortmund, Deutschland

joachim.scheiner@tu-dortmund.de

(c) (1) () 2021 Seyfarth; licensee oekom verlag. This Open Access article is published under a Creative Commons Attribution 4.0 International License. während fast die Hälfte eher suburban-kleinstädtische Vorstellungen hat und mit 28 Prozent ein substanzieller Anteil geradezu antiurban eingestellt ist. Diese Präferenzen werden zweitens beeinflusst von den Wohnerfahrungen der Befragten in der Kindheit und Jugend, von intergenerationalen Effekten (Wohnumfeldwünsche und Mobilitätseinstellungen der Eltern), den eigenen Mobilitätseinstellungen und der realisierten Alltagsmobilität. Im Hinblick auf die künftige Raumentwicklung ergibt sich in der Konsequenz, dass Reurbanisierung keinesfalls als Selbstläufer angesehen werden kann.

Schlüsselwörter: Reurbanisierung - Suburbanisierung Stadtentwicklung - Wohnpräferenzen - Wohnwünsche Studierende $=$ Wohnbiografie

Really permanently urban? An empirical Study of Young Adults' Short- and Long-term Wishes for Residential Environment as a Contribution to the Debate on Reurbanization

\section{Abstract}

The residential migrations and housing preferences of young adults play a significant role in the context of the reurbanization debate. They are regularly identified as a main contributor group to reurbanization. In this context, students are of particular importance for many cities. However, the findings mainly refer to Generation Y (born circa 1980-2000). Regarding the subsequent Generation Z ("post-millennials"), less is known. One way to understand the dynamics and direction of possible changing housing environment desires is to ask young adults about how they would like to live after completing their education or later. This paper analyses the housing aspirations of a sample of spatial planning students in Dortmund. To under- 
stand the factors influencing housing desires a multinomial logit model is estimated. The main results show, firstly, that only very few students want to live urban in the long term, while almost half have rather suburban and small town preferences. A substantial share (28 percent) is even anti-urban. Second, these preferences are determined by the respondents housing experiences in childhood and adolescence, by intergenerational effects (parents housing preferences and mobility attitudes), by individual mobility attitudes, and by realized everyday mobility. With regard to future spatial development, it can be concluded that reurbanization can by no means be regarded as a self-propelling process.

Keywords: Reurbanization - suburbanization - urban development - housing preferences - housing wishes students = housing biography

\section{Einleitung}

Die Reurbanisierung bestimmt seit der einflussreichen Studie von Brühl, Echter, Frölich von Bodelschwingh et al. (2005) und dem von Brake und Herfert (2012) vorgelegten Sammelband die planungswissenschaftliche und praktische Debatte um aktuelle Trends der Raumentwicklung. Damit ist sie eines der derzeit meistdiskutierten Themenfelder der Stadtforschung (vgl. Engler 2014: 9; Siedentop 2018: 381; Dembski/Sykes/Couch et al. 2019: 1). In der außerfachlichen Öffentlichkeit wird dies breit, aber widersprüchlich wahrgenommen, wie sich an Schlagzeilen wie diesen zeigt: „Dorfbewohner kämpfen gegen Landflucht“ (Gebert 2017) vs. „Jetzt geht es wieder raus aufs Land“ (Ochs 2016).

Die wachsende Aufmerksamkeit für Reurbanisierung wird begleitet von einer definitorischen Unbestimmtheit sowie empirischen Offenheit des Begriffs (vgl. Hesse 2010: 44). Reurbanisierung zeigt zahlreiche Facetten der unterschiedlichen Dimensionen Wirtschaft, Demographie und Sozialstruktur und lässt sich qualitativ und quantitativ mit vielfältigen Methoden bestimmen (Brake/Urbanczyk 2012). Unbestritten ist, dass es eine Vielzahl von empirischen Belegen für ein demographisches Wachstum der (meisten) großen Städte gibt, auch wenn das Bild im Einzelfall differenziert ist (Herfert/Osterhage 2012; Milbert/Sturm 2016; Dembski/Sykes/Couch et al. 2019) und bereits der Sammelband von Brake und Herfert (2012) die Parallelität von Sub- und Reurbanisierungsprozessen zeigt. Seit etwa zehn Jahren befinden sich viele Groß- und Universitätsstädte in Deutschland in einer Wachstumsphase (vgl. Milbert 2017: 3-8; Held/Mäding 2020: 29). Gleichzeitig mehren sich die Anzeichen dafür, dass sich das Wachstum aufgrund zunehmend beschränkter Wohnungsmärkte bzw. Flächenreserven verlangsamt und möglicherweise gerade in den attraktiven Stadtregionen zugunsten peripherer Lagen im suburbanen Umland umkehrt (Busch 2016; Milbert 2017). Busch (2016) verdeutlicht, dass bereits zwischen 2008 und 2014 - auf dem Höhepunkt der planerischen Reurbanisierungs-Euphorie - viele Großstädte starke Rückgänge des Wanderungssaldos verzeichneten (wenn die Auslandszuwanderung ausgeklammert wurde), aus der sich das Wachstum der Städte in erheblichem Maße speist. Bei den in diesen Arbeiten vorgenommenen Datenanalysen werden verschiedene Komponenten der Bevölkerungsentwicklung und des Wanderungsgeschehens betrachtet. Daraus resultierend kann aufgedeckt werden, dass die längere Phase der Reurbanisierung mehrere Abschnitte mit unterschiedlichen Treibern umfasst.

Die Wanderungen und Wohnbedürfnisse junger Erwachsener spielen in diesem Zusammenhang eine bedeutende Rolle. Regelmäßig werden sie in ihrer Funktion als Bildungswanderer als eine Hauptträgergruppe der Reurbanisierung identifiziert (vgl. Köppen 2008: 35; Engler 2014: 16-17; Matthes 2016: 100; ARL 2018: 2; Scholich 2019: 8). Dabei spielen Studierende mengenmäßig eine große Rolle. Daneben sind Studierende auch ,qualitativ ‘ für die Städte von großer Bedeutung, weil sie als hochqualifizierte Absolventinnen und Absolventen häufig in den Städten verbleiben und dort Familien gründen, während in früheren Generationen gerade die Familiengründungsphase als ein Moment der Abwanderung in das suburbane Einfamilienhaus mit Garten und (zwei) Pkw typisch war. Dieser Befund erscheint wegweisend für die Antizipation künftiger Stadtentwicklung: Junge Familien verlassen nicht mehr ,automatisch ' die Städte zugunsten eines Eigenheims im Grünen, sondern bleiben häufiger dort, als dies vor Jahrzehnten der Fall war (Herfert/ Osterhage 2012: 109; Milbert/Sturm 2016: 141-142), teils um sich dort in einer ,,inneren Suburbanisierung“ einzurichten (Frank 2018).

Die hier angesprochenen Befunde beziehen sich im Wesentlichen auf die Generation Y (geboren etwa 19802000, auch Millennials genannt; vgl. Hopkins/Stephenson 2014: 88), die inzwischen teilweise Familien gegründet hat. Über die nachfolgende Generation Z (auch Post-Millennials) ist allerdings weniger bekannt, weil ihre Erwartungen, Wünsche und Hoffnungen für ihren späteren Lebenslauf in Bezug auf Wohnen, Mobilität, Familie oder Karriere noch nicht anhand faktischer Entscheidungen verfolgt werden können. Nach der jüngsten Shell-Jugendstudie (Albert/ Hurrelmann/Quenzel et al. 2019) lässt sich die Generation Z (hier: geboren 1996-2007) nur in Teilen als ähnlich der Generation Y charakterisieren. Geblieben ist die pragmatische Grundorientierung, der Leistungswille, Achtsamkeit in der eigenen Lebensführung (z. B. Work-Life-Balance) und der Wunsch nach stabilen Beziehungen im nahen Umfeld (Familie, Partnerschaft). Deutlich zugenommen 
hat dagegen das politische Interesse, der Stellenwert von Umwelt- und Klimaschutz, der Wunsch nach mehr Mitsprache, der ausgeprägte Sinn für Gerechtigkeit und die grundsätzliche Zufriedenheit mit der Demokratie als Staatsform. Gleichzeitig ist der Sinn für Hedonismus zunehmend stark ausgeprägt. Bei beiden Geschlechtern ist ein gewisser Konservatismus in den Vorstellungen von Geschlechterrollen erkennbar. Aus räumlicher Perspektive ist zu ergänzen, dass diese Generation in einer Welt aufgewachsen ist, in der das Auto und der suburbane Raum in selbstverständlicher Weise allgegenwärtig sind.

Eine Möglichkeit zum Verständnis der Dynamik und Richtung sich möglicherweise verändernder Wohnumfeldwünsche besteht darin, junge Erwachsene danach zu fragen, wie sie nach Beendigung der Ausbildung oder später wohnen möchten (vgl. von Einem 2016: 152; ähnlich Kramer und Pfaffenbach 2016 für ältere Menschen). Ähnlich wird dies auch über Vorstellungen und Wünsche in Bezug auf künftige Mobilität und weitere Lebensbereiche gemacht (Delbosc/Naznin 2019). Dies erlaubt keine ,harte' Prognose, weil für tatsächliche zukünftige Entscheidungen nicht nur die Wünsche, die jemand im jungen Erwachsenenalter hat, entscheidend sind, sondern viele andere Faktoren eine Rolle spielen. Es gestattet aber einen Blick auf die Präferenzen und Vorstellungen, die junge Erwachsene von ihrer Zukunft haben, und damit eine Einschätzung, inwieweit städtisches Wohnen aus der Sicht der jungen Generation ein individuell dauerhaftes Phänomen sein könnte oder ob dies eher als kurze Lebensphase gesehen wird. Wichtig ist es festzuhalten, dass diese Perspektive ein ,Schnappschuss" ist. Wünsche ändern sich im Lebensverlauf, auch beeinflusst durch Rahmenbedingungen (Immobilienmärkte, Lebenssituationen).

In diesem Beitrag wird der Frage nachgegangen, inwieweit es sich beim städtischen Wohnen junger Erwachsener in deren eigener Wahrnehmung um eine gefestigte Präferenz handelt, die als stabil angesehen wird - oder eher um einen zeitlich begrenzten Lebensabschnitt, dem eher suburban-periphere Wohnumfeldwünsche für die Zeit nach Beendigung der Ausbildungsphase gegenüberstehen. Hinsichtlich der facettenreichen Reurbanisierungsdebatte untersuchen wir die individuellen Vorlieben, die einer tatsächlichen Wohnstandortentscheidung vorgelagert sind. Damit ordnet sich die Untersuchung definitorisch in das Feld der Arbeiten zum Thema Reurbanisierung ein, die sich aus einer analytischquantitativen Perspektive der Funktion des Wohnens widmen. Demnach würde eine dominierende Präferenz unter jungen Erwachsenen zugunsten eines städtisch geprägten Wohnumfelds für einen zukünftig starken Reurbanisierungstrend sprechen.

Für die Modellierung der Wohnumfeldwünsche greifen wir auf eine Reihe von Parametern zurück, die einen $\mathrm{Zu}$ - sammenhang mit den Wünschen vermuten lassen, nämlich Mobilitätseinstellungen, Verkehrsmittelnutzung, intergenerationale Einflüsse (Wohnumfeldwünsche und Mobilitätseinstellungen der Eltern) und Wohnerfahrungen in der Kindheit und Jugend. Wir verwenden dazu einen Datensatz der TU Dortmund. Studierende der Raumplanung, deren Eltern und zwei ihrer Großeltern waren zu wohn- und mobilitätsbezogenen Themen befragt worden. Wir konzentrieren uns auf die Wohnumfeldwünsche der Studierenden, die zum Befragungszeitpunkt am Ende des ersten Semesters sind und betrachten diese Wünsche kombiniert in kurz-, mittel- und langfristiger Perspektive. Dies sind schwerpunktmäßig die Geburtsjahrgänge 1996-1998, die als frühe Kohorten der Generation Z gelten können.

In Kapitel 2 wird der derzeitige Forschungsstand zur Rolle von jungen Erwachsenen in der Reurbanisierungsdebatte sowie ihre Wohnumfeldwünsche und Wohnstandortwahl in dieser Lebensphase umrissen, um dann die Bedeutung von Wohnbiographien und Sozialisation für zukünftige Wohnvorstellungen aufzuzeigen. Im methodischen Teil (Kapitel 3) folgen Informationen zum verwendeten Datensatz und zu den Variablen, die in das multinomiale Logitmodell eingeflossen sind. In Kapitel 4 wird zunächst die Verteilung der Wohnumfeldwünsche unter den Studierenden kurz beschrieben, bevor die Ergebnisse der Modellschätzung zu den Einflussfaktoren vorgestellt und diskutiert werden. Der Beitrag schließt mit Überlegungen ab, welche Schlussfolgerungen aus den neuen Erkenntnissen zu den mittel- und langfristigen Wohnumfeldwünschen von jungen Erwachsenen der Generation Z im Hinblick auf die Dauerhaftigkeit des in den letzten Jahren zu beobachtenden Reurbanisierungstrends gezogen werden können (Kapitel 5).

\section{Stand der Forschung}

\subsection{Junge Erwachsene in der Reurbanisierungsdebatte}

Bei der Trendverschiebung in Richtung Reurbanisierung, die in Deutschland in den Jahren nach der Jahrtausendwende einsetzte, spielen junge Erwachsene eine zentrale Rolle. Zwar wird einerseits betont, dass sich diese Entwicklung - etwa im Gegensatz zur Gentrifizierung - auf ein breites Spektrum an sozialen und demographischen Gruppen stützt (Haase/Herfert/Kabisch et al. 2010: 28; Siedentop/ Zakrzewski/Stroms 2018: 4). Andererseits werden jedoch junge Erwachsene immer wieder als wichtige Trägergruppe identifiziert, wenn es um das neue Wachstum von Städten und urbanen Quartieren geht (Kabisch/Steinführer/Haase 2012: 122-125).

Die zentrale Bedeutung der jungen Erwachsenen für den 
Reurbanisierungstrend wird in Analysen zum Wanderungsgeschehen besonders deutlich. Bereits in der Vergangenheit hat sich in vielen Studien gezeigt, dass Altersgruppen charakteristische räumliche Wanderungsmuster aufweisen. Junge Erwachsene ziehen bevorzugt in Städte, was dort zu Wanderungsgewinnen bei den entsprechenden Jahrgängen führt (Bucher/Schlömer 2012: 67). Dieses Plus der Kernstädte in Agglomerationsräumen und im übrigen Bundesgebiet hat sich insbesondere in der Altersgruppe der 18- bis 24-Jährigen mit Beginn der 2000er-Jahre noch einmal erheblich vergrößert, während agglomerationsferne Räume und die Umlandgemeinden eine Zunahme der Wanderungsverluste bei Menschen in diesem Alter erfahren haben (Sander 2014: 229-230).

Dass junge Erwachsene bevorzugt in innenstadtnahe Quartiere von größeren Städten ziehen, ist zwar seit langer Zeit bekannt, hat aber offenbar eine neue Dimension erreicht. Zunächst für Länder aus dem angelsächsischen Raum beschrieben, wird diese Entwicklung mittlerweile auch hierzulande diskutiert. Im Rahmen einer Studie für kanadische Großstädte hat Moos (2016) den Begriff der „youthification“ eingeführt. Ebenso kursiert das Schlagwort der „studentification“, wobei - anknüpfend an Debatten zur Gentrifizierung - damit verstärkt auf Aspekte der Überformung und Verdrängung eingegangen wird (Smith 2005; Hubbard 2009). Siedentop, Zakrzewski und Stroms (2018) erkennen auch für deutsche Stadtregionen eine zunehmende Segregation nach Altersgruppen und betrachten die Konzentration von jungen Menschen in den zentralen Wohngebieten explizit im Zusammenhang mit dem Reurbanisierungstrend der letzten Jahre. Auch die Überlegungen rund um den Begriff der ,Schwarmstädte“ (Simons/Weiden 2016: 263), die hierzulande eine große Popularität erfahren haben, zielen in eine ähnliche Richtung. Demnach entspricht die Reurbanisierung einer Konzentration von jungen Menschen auf einige ausgesuchte Städte, die neben der Ausbildungswanderung auch durch die Berufsanfängerwanderung herbeigeführt wird.

Bezogen auf die Ursachen für die durch junge Erwachsene getragene Reurbanisierung werden verschiedene Erklärungsansätze vorgebracht. Zuallererst werden die Expansion des Bildungswesens und die Akademisierung von Ausbildung als wesentliche Treiber angesehen (Siebel 2010: 106). Durch diese Entwicklungen treibt es die jungen Erwachsenen noch einmal stärker in die Städte als in der Vergangenheit. Teilweise wird zudem auf einen Mengeneffekt hingewiesen, der auf vergleichsweise geburtenstarke Jahrgänge zurückzuführen ist. Bereits in den 1980er-Jahren hat die Babyboomer-Generation in Deutschland (geboren 1955-1969) für eine kurze Phase der Reurbanisierung gesorgt, als diese in die Lebensphase der Ausbildung und des Berufseinstiegs eingetreten ist. Nach dem Beginn des neuen Jahrhunderts sind es dann die Kinder dieser Generation, die es in großer Zahl in die Hochschulstädte und in andere Zentren zieht (Bucher/Schlömer 2012: 67). Auch der Schwarmstadt-Ansatz geht von veränderten Rahmenbedingungen aus, stellt jedoch die, langfristig betrachtet, seit mehreren Jahrzehnten rückläufigen Geburtenzahlen in den Mittelpunkt. Da junge Menschen zu einer Minderheit werden, sind sie gewissermaßen dazu gezwungen, sich an bestimmten Orten zu konzentrieren, um ihre altersspezifischen Standortpräferenzen und generationale Identität realisieren zu können (Simons/ Weiden 2016: 267-269).

\subsection{Wohnumfeldwünsche und Wohnstandortwahl junger Erwachsener}

Ebenso wie es beim Wanderungsgeschehen charakteristische Muster gibt, bestehen auch relativ klare Unterschiede zwischen Altersgruppen bei den für eine Wohnstandortwahl relevanten Kriterien. In Wanderungsmotivuntersuchungen zeigt sich, dass bei der Suche nach einem Standort Wohnwünsche und Bedürfnisse auf bestehende Restriktionen wie die Verfügbarkeit bestimmter Angebote und das vorherrschende Preisniveau treffen (aktuell z. B. Stadt Dortmund 2020: 17; Stadt Wolfsburg 2020: 65). Im Ergebnis sind für viele junge Menschen eine bezahlbare Wohnung, die Anbindung an den öffentlichen Personennahverkehr (ÖPNV) und die Nähe zu Freunden von besonderer Bedeutung (DittrichWesbuer/Osterhage 2008; Kühl 2014; Thomas/Serwicka/ Swinney 2015). Für Studierende ist weiterhin die Nähe zur Hochschule bei der Wahl des Wohnstandortes überaus wichtig, was insgesamt das Vorherrschen einer ausgeprägten Distanzempfindlichkeit in der Alltagsmobilität unterstreicht (Münter/Osterhage 2017: 6-7). Ähnliche Punkte werden von Kramer (2019: 285) aufgelistet, die bei Studierenden von spezifischen Anforderungen an ein Quartier wie sichere Fahrradwege, große Nähe und nächtliche Erreichbarkeit des ÖPNV ausgeht. Simons und Weiden (2016: 264-269) begründen das auf ausgewählte Städte ausgerichtete Schwarmverhalten von jüngeren Menschen - gemeint sind in diesem Fall 15- bis 34-Jährige - vor allem über den Wunsch nach Nähe zu Gleichaltrigen und das Vorhandensein von Kristallisationspunkten des öffentlichen Lebens.

Klassische Theorien wie auch neuere Beiträge der Wanderungsforschung betonen die Bedeutung, die der Stellung von Wohnumfeldwünschen und Wohnstandortwahl im Lebensverlauf zukommt (Findlay/McCollum/Coulter et al. 2015; Coulter/van Ham/Findlay 2016). Zugleich unterstreichen empirische Ergebnisse aus der Lebenslaufforschung, dass Wanderungen in bestimmte Richtungen im Zusammenhang mit beruflichen und familialen Ereignissen stehen. Dazu passen Untersuchungen, die die Relevanz von Rückwanderungen von jungen Menschen aus städtischen 
Räumen in ländliche Gebiete nach der Ausbildung oder dem Berufseinstieg herausstellen (Fuchs/Nadler/Roth et al. 2017). Allerdings zeichnen sich mit dem Trend zur Reurbanisierung interessante Verschiebungen bei den lange Zeit vorherrschenden Mustern ab: Nach den Ergebnissen von Gerber (2011: 277-278) wird in den jüngeren Geburtskohorten zum einen vermehrt mit Bildungs- bzw. Erwerbsaufnahme in Kernstädte gewandert, gleichzeitig gehen zum anderen (nicht-)eheliche Partnerschaften und bevorstehende Geburten seltener mit Fortzügen ins Umland einher. Zudem kann beobachtet werden, dass Familien eine Wohnsituation und ein Wohnumfeld nach ihren Bedürfnissen schaffen, um über die Ausbildungs- und Berufseinstiegsphase hinaus an städtischen Standorten zu wohnen, ohne auf die Vorzüge des suburbanen Lebens zu verzichten (Karsten 2003; Frank 2018). Zeitweise war dies durch die Mobilisierung von Gewerbe- und Bahnbrachen in vielen Städten möglich; inzwischen sind diese Reserven in vielen Städten zunehmend aufgebraucht (vgl. Brombach/Jessen 2017).

Im Hinblick auf die Dauer des Reurbanisierungstrends besteht somit eine entscheidende Frage darin, welche Wohnstandorte die heutige Gruppe der jungen Erwachsenen in zukünftigen Lebensphasen bevorzugt. Kleinere experimentelle Umfragen können hierzu erste hilfreiche Hinweise geben. Dies gilt zum Beispiel für eine Befragung von Studierenden wohnungswirtschaftlicher Seminare in Berlin, die sich zu ihren Wohnvorstellungen zehn Jahre nach dem Berufseinstieg äußern sollten (von Einem 2016: 152-155). Die Ergebnisse sprechen dafür, dass traditionelle Wohn- und Lebensformen als Zukunftsbild weiterhin eine große Bedeutung aufweisen. Der Wunsch nach einem freistehenden Haus am Stadtrand oder im Umland ist weit verbreitet wenn auch möglicherweise nicht mehr so dominant wie bei früheren Generationen. Ganz ähnliche Schlussfolgerungen lassen sich aus einer Untersuchung von Weller (2016) unter Studierenden in Karlsruhe ableiten, bei der eine Mehrheit der jungen Erwachsenen anhand fingierter Immobilienanzeigen ein „Häuschen im Grünen“ für die Zukunft favorisiert hat. Diese Befunde zu den Vorstellungen der Studierenden unter den jungen Erwachsenen leisten einen wichtigen Beitrag zum Verstehen der künftigen Wohnungsnachfrage von gut verdienenden Haushalten, zu denen sie in der Regel zukünftig gehören (Glatter/Hackenberg/Wolff 2014; von Einem 2016: 152). Aus diesen Studien liegen jedoch keine Erkenntnisse darüber vor, von welchen Einflussgrößen die zukünftigen Wohnvorstellungen und Wohnumfeldwünsche abhängen. Zudem sagen diese Untersuchungen bisher kaum etwas über mögliche zeitliche Differenzierungen von Wohnvorstellungen über den Lebenslauf aus.

\subsection{Die Bedeutung von Wohnbiographien und Sozialisation}

An dieser Stelle ergeben sich weiterführende Erkenntnisse aus Längsschnittstudien, die den Einfluss von Wohnbiographien auf spätere Wohnstandortentscheidungen untersuchen. Basierend auf Mulder und Hooimeijer (1999) kann davon ausgegangen werden, dass individuelle Wohnerfahrungen auf zukünftige Wohnstandortentscheidungen einwirken. Gemäß einem handlungstheoretischen Erklärungsansatz greifen Individuen auf bereits gesammelte Erfahrungen zurück, um die eigenen Bedürfnisse und Vorstellungen bestmöglich zu befriedigen (Albrecht 2015: 243). Ob ein urbanes, suburbanes oder ländliches Wohnumfeld gewählt wird, hängt nach vorliegenden Forschungsergebnissen davon ab, welche Wohnerfahrungen insbesondere in der eigenen Kindheit gesammelt wurden (Aero 2006; Albrecht/ Döring/Holz-Rau et al. 2019). Für die Niederlande konnten in einer Studie hierzu differenzierte Befunde herausgearbeitet werden: Demnach führen Wohnerfahrungen in Städten und in Vororten vor allem zu dem Wunsch, später an genau diese Wohnorte zurückzuwandern. Dagegen ergibt sich aus frühen suburbanen und ländlichen Wohnerfahrungen häufig eine allgemeine Vorliebe für ländliche Gegenden bei Wohnstandortentscheidungen (Feijten/Hooimeijer/Mulder 2008).

Wohnbiographien sind als Teil individueller Lebensläufe nicht isoliert von Lebensläufen anderer Personen zu betrachten, sondern ,eingebettet in soziale Strukturen auf der persönlichen Ebene, in familiäre, freundschaftliche oder nachbarschaftliche Netzwerke“ (Holz-Rau/Scheiner 2015: 13). Der Einfluss von Personen aus dem engeren Umfeld oder dem eigenen Haushalt in unterschiedlichen Lebens- und Entscheidungsphasen kann als Sozialisationseffekt bezeichnet werden (Döring 2015: 35-37). Neben der Schule und dem Freundeskreis spielen vor allem Eltern durch den engen Kontakt und ihre Vorbildfunktion gegenüber den Kindern als Sozialisationsinstanz eine bedeutende Rolle (vgl. Hurrelmann 2012: 133; Döring 2015: 24). Für die Übertragung von Verhaltensweisen der Eltern auf ihre Kinder in Bezug auf die Wohnmobilität gibt es bereits einige empirische Belege (Myers 1999: 877-879; Blaauboer 2011: 1644-1647). Eltern können sowohl direkten Einfluss auf Wohnstandortpräferenzen und -entscheidungen ihres Kindes nehmen, indem sie an der Entscheidungsfindung beteiligt werden, als auch indirekten Einfluss durch die Weitergabe von Werten, Normen oder Einstellungen ausüben (Albrecht 2015: 245). Darüber hinaus wirken auch die Wohnstandorte von Familienmitgliedern außerhalb des Haushalts - vor allem Großeltern und Geschwister - im Sinne einer kontinuierlichen Sozialisation auf die eigene Wohnstandortwahl ein, was nach den Ergebnissen von statistischen Analysen zu großen Ähnlichkeiten beim gewählten Um- 
feld führt (Blaauboer 2011). Die Sozialisation kann hierbei aber durchaus unterschiedliche Formen annehmen. Während die Reurbanisierung junger Menschen im Nordamerika der 1980er-Jahre eher den Charakter einer Rebellion gegen die elterlichen Vorstellungen eines, guten Lebens“ im suburbanen Eigenheim mit Garten und mehreren Pkw hatte (Caulfield 1994), deuten andere Befunde eher auf positive Korrelationen zwischen den Wohnstandortentscheidungen zweier Generationen einer Familie hin (Blaauboer 2011).

Wenig Wissen gibt es bislang über die sozialisatorischen Einflüsse der Mobilität im Alltag. Es ist allerdings bekannt, dass die Wohnstandortwahl von Haushalten von deren PkwBesitz abhängt (Hesse/Scheiner 2009). Es kann zumindest vermutet werden, dass die Sozialisation mit bestimmten Verkehrsmitteln und Routinen der Alltagsmobilität sowie die Einstellungen zu Verkehrsmitteln (z. B. Auto-Affinität) auch für Wohnstandortentscheidungen eine wichtige Rolle spielen. Für beides - Verkehrsmitteleinstellungen und Verkehrsmittelnutzung - wurden in ersten Studien auch positive Korrelationen zwischen zwei Generationen einer Familie gefunden (Döring/Albrecht/Scheiner et al. 2014).

\section{Daten und Methodik}

\subsection{Datengrundlage}

Gegenstand der Untersuchung sind die gegenwärtigen und zukünftigen Wohnumfeldwünsche von Studierenden. Nach den Ausführungen zum hier dargestellten Stand der Forschung besteht die weit verbreitete Überzeugung, dass diese Gruppe in ihrer gegenwärtigen Lebensphase eine starke Präferenz zugunsten städtischer Wohnstandorte aufweist und damit als wesentliche Trägerin des Reurbanisierungstrends der letzten Jahre anzusehen ist. Es lassen sich aber nur wenige Anhaltspunkte finden über die Vorstellungen und Wünsche, die die jungen Erwachsenen der sogenannten Generation $\mathrm{Z}$ im Hinblick auf ihre Wohnstandorte im weiteren Lebensverlauf haben. Es geht also um die Frage, ob sie - im Gegensatz zu früheren Generationen - dauerhaft an urban geprägten Standorten leben möchten oder doch andere räumliche Optionen bevorzugen. Hierbei sollen zudem mögliche Einflussfaktoren auf die zukünftigen Vorstellungen beleuchtet werden.

Die für eine solche Analyse benötigten Variablen stehen in keinem vorhandenen Datensatz (z. B. Sozioökonomisches Panel) simultan zur Verfügung. Für die empirische Studie greifen wir deshalb auf einen Datensatz der Technischen Universität Dortmund zurück, der in den Jahren 2015, 2016 und 2017 im Rahmen von Übungslehrveranstaltungen der Fakultät Raumplanung erhoben wurde. Es handelt sich um eine wiederholte retrospektive Querschnitts- erhebung, nicht um ein Panel. Der Fragebogen ist eine stark überarbeitete Fassung einer Vorgängerversion, die für das Projekt „Generationsübergreifende Mobilitätsbiografien“ verwendet wurde (vgl. Scheiner/Sicks/Holz-Rau 2014).

Der Fragebogen wird von den Studierenden, die zum Befragungszeitpunkt am Ende des ersten Semesters sind, nach einer kurzen Schulung selbst ausgefüllt. Die Studierenden erhalten den Auftrag, ihrerseits ihre Eltern und zwei ihrer Großeltern (je ein Großelternteil mütter- und väterlicherseits) zu befragen. Die Teilnahme ist verpflichtend. Die Studierenden haben allerdings individuell die Möglichkeit, ihre ausgefüllten Fragebögen lediglich zur Kontrolle vorzuzeigen, ohne sie abzugeben. Von dieser Möglichkeit machen erfahrungsgemäß null bis zwei Studierende je Jahrgang Gebrauch.

Der Datensatz umfasst 586 Studierende, 1052 Elternteile und 827 Großelternteile. Darüber hinaus wurden 256 sonstige Personen erfasst, deren Befragung den Studierenden freistand, wenn ein Teil der Familienmitglieder nicht befragt werden konnte. Die Fälle der hier dargestellten Regressionsanalyse sind die Studierenden, für die vollständige Informationen vorliegen. Dies schließt insbesondere die verwendeten Variablen für beide Elternteile ein. Stiefelternteile werden als Eltern behandelt. Studierende, die keinen Elternteil befragt haben, werden aus der Analyse ausgeschlossen. Die Nettostichprobe im Regressionsmodell umfasst $n=552$ Studierende.

\subsection{Fragebogen}

Der Fragebogen enthält Fragen zu wohn- und mobilitätsbezogenen Themen in einer Lebenslaufperspektive (retrospektiv und in geringerem Umfang auch prospektiv für unterschiedliche Zeitpunkte bzw. Phasen im Leben). Dies und die Möglichkeit der Verknüpfung mehrerer Generationen einer Familie machen den Datensatz nach unserem Wissen einzigartig. Wir konzentrieren uns auf die Wohnumfeldwünsche der Studierenden. Diese Wünsche werden für vier Zeithorizonte im Lebensverlauf erhoben: jetzt; in fünf bis zehn Jahren (also typischerweise in den ersten Berufsjahren); längerfristig; nie. Auch die unbestimmte Antwort „weiß nicht“ ist möglich. Wir kombinieren diese Fristigkeiten in eine einzige Variable, die damit kurz-, mittelund langfristige Wohnumfeldwünsche einschließlich unbestimmter Wünsche gemeinsam abbildet.

Die Frageformulierung lautet ,Wie möchten Sie wohnen?" mit der Nennung der Fristigkeiten in vier Spalten (vgl. Tabelle 1). Die Frage bezieht sich auf sechs verschiedene inhaltliche Kategorien (in Zeilen), nämlich folgende vier Lagetypen: 
Tabelle 1 Zuordnung der Studierenden zu den fünf Ausprägungen anhand der Frage „Wie möchten Sie wohnen?“ (vereinfachte Darstellung)

\begin{tabular}{|c|c|c|c|c|}
\hline & jetzt & in 5-10 Jahren & längerfristig & nie \\
\hline Urban & & (I) & 1 & \\
\hline Antiurban & $\mathrm{SR} / \mathrm{KM} / \mathrm{L} / \mathrm{G}$ & $\mathrm{SR} / \mathrm{KM} / \mathrm{L} / \mathrm{G}$ & $\mathrm{SR} / \mathrm{KM} / \mathrm{L} / \mathrm{G}$ & $I^{*}$ \\
\hline Suburban/Kleinstädtisch & & (SR / KM) & $\mathrm{SR} / \mathrm{KM}$ & \\
\hline Ländlich & & (L) & $\mathrm{L}$ & \\
\hline Offen & & w.n. / k.A. & w.n. / k.A. & nicht I \\
\hline
\end{tabular}

$\mathrm{I}=$ Innenstadt, $\mathrm{SR}=$ Stadtrand, $\mathrm{KM}=$ kleine/mittelgroße Stadt, $\mathrm{L}=$ Land $/$ Dorf, $\mathrm{G}=$ Haus $/$ Wohnung mit Garten

w.n. = weiß nicht, k.A. = keine Angabe

$(. .)=$. sofern kein längerfristiger Wohnumfeldwunsch

* = Die Zuordnung zur Gruppe „Antiurban“ erfolgt entweder durch die Angabe SR / KM / L / G in den drei Zeitfenstern oder durch die Angabe „nie Innenstadt“

- in der Innenstadt einer Großstadt

- am Stadtrand einer Großstadt

- in einer kleinen oder mittelgroßen Stadt

- auf dem Land/Dorf

und zwei Kategorien des direkten Umfelds und des Rechtsverhältnisses:

- in einem Haus/einer Wohnung mit Garten

- im Eigentum (Haus/Wohnung)

Der Eigentumswunsch wird in unserer Analyse nicht berücksichtigt, weil er zwar Treiber der Randwanderung sein kann, in sich aber keine standortbezogene Aussage darstellt.

\subsection{Zielvariable: Wohnumfeldwünsche im weiteren Lebensverlauf}

Die Daten lassen vielfältige Kombinationen von Wohnumfeldwünschen in unterschiedlichen Fristen zu. Unsere Zielvariable ist deshalb eine Kombination der Wünsche der jungen Studierenden (Lagetyp und Garten ja/nein) für die vier genannten Zeithorizonte. Es ist $\mathrm{zu}$ beachten, dass die Befragten je Zeithorizont mehrere positive Antworten geben konnten. Um Regelmäßigkeiten und typische Kombinationen von Antworten zu ermitteln, wurden zahlreiche Kreuztabellen unter Beachtung von Mehrfachantworten erstellt. So nennen beispielsweise 57 Studierende die Kombination ,,jetzt: Innenstadt“, ,längerfristig: Stadtrand“ und ,nie: Land".

Die Zuordnung der Studierenden zu einer Gruppe wurde aufgrund der Fülle möglicher Antwortkombinationen manuell vorgenommen. Dabei wird den längerfristigen Wünschen stärkeres Gewicht verliehen, da diese den längsten Zeitabschnitt umfassen und für die Frage nach der Dauerhaftigkeit städtischer Wohnumfeldwünsche über die Phase des Studiums hinaus wichtig sind. Die endgültige Variable durchlief eine längere Entwicklungsphase, wobei zunächst mit stark differenzierten Gruppen gearbeitet wurde. Diese wurden im weiteren Verlauf zusammengefasst, weil sich die Gruppengrößen als zu gering erwiesen, um ein statistisch bedeutsames Modell zu erzielen, und die Wohnumfeldwünsche unterschiedlicher Gruppen teilweise deutliche Ähnlichkeiten aufwiesen.

Letztlich werden hier fünf Ausprägungen unterschieden: „Urban“, „Antiurban“, „Suburban/Kleinstädtisch“, „Ländlich“ und „Offen“. Die Zuordnungsregel wird im Folgenden und in Tabelle 1 dargestellt. Die Gruppe „Urban“ umfasst Studierende mit einem langfristigen Wunsch: Innenstadt einer Großstadt (wobei gleichzeitig auch andere Lagetypen gewünscht sein können). Die Gruppe „Antiurban“ vereint Studierende, die nie bzw. in keinem möglichen Zeitfenster in der Innenstadt einer Großstadt wohnen wollen. Die Gruppe „Suburban/Kleinstädtisch“ subsumiert Studierende mit einem längerfristigen Wohnumfeldwunsch am Stadtrand einer Großstadt oder in einer kleinen bis mittelgroßen Stadt, unabhängig davon, wie die kurz- bis mittelfristigen Wünsche aussehen. Sofern keine Angabe über einen längerfristigen Wohnumfeldwunsch vorlag, wurde der mittelfristige Wunsch für die Gruppenzugehörigkeit herangezogen. Die beiden Ausprägungen ,,am Stadtrand einer Großstadt“ und ,in einer kleinen bis mittelgroßen Stadt“" werden in einer Gruppe vereint, da eine nicht unerhebliche Anzahl Studierender beide Raumkategorien (Stadtrand oder kleine mittelgroße Stadt) längerfristig bevorzugt und diese demgemäß nicht eindeutig zu differenzieren sind. Die Ausprägung „Ländlich“ führt alle Fälle mit einem längerfristig ländlichen Wohnumfeldwunsch zusammen, wieder unabhängig von den kurz- bis mittelfristigen Wünschen. Liegt kein langfristiger Wohnumfeldwunsch vor, wird auch hier stellvertretend der mittelfristige Wunsch herangezogen.

Für eine ergänzende Klassifikation wird zudem der Wunsch nach einem Garten herangezogen, und zwar ausschließlich für Befragte, die längerfristig keine Präferenz für einen bestimmten Raumtyp äußern. Befragte mit der Kombination ,längerfristig: mit Garten“ und „nie: auf dem 
Land/Dorf“" werden der Gruppe „Suburban/Kleinstädtisch“ zugeordnet, wenn ansonsten keine eindeutige Zuordnung möglich wäre, weil der Garten eine Präferenz für geringe Dichte ausdrückt und demnach gegen Urbanität spricht. Dem folgend wird die Kombination ,längerfristig: mit Garten“ und „nie: Suburban/Kleinstädtisch“ der Gruppe „Land/ Dorf" zugeordnet. Damit wird die unspezifische Gruppe „Offen“ deutlich verkleinert (von 100 auf 54 Fälle). Die Gruppe „Offen“ umfasst Fälle, die durch das Raster der hier beschriebenen Klassifikation fallen. Insgesamt umfasst die verwendete Stichprobe danach $n=552$ Fälle.

Es wird darauf hingewiesen, dass die Gruppe „Urban“ trotz der kleinen Gruppengröße beibehalten wird, weil sie für die inhaltliche Fragestellung des vorliegenden Beitrags zentrale Bedeutung besitzt.

\subsection{Weitere Variablen}

\subsubsection{Merkmale der Studierenden}

Verkehrsmitteleinstellungen der Studierenden wurden auf fünfstufigen Likert-Skalen erhoben. Sie werden hier als metrisch betrachtet, weil es keinen Anlass zur Vermutung gibt, dass die Skala nicht äquidistant sein könnte. Die Einstellung gegenüber öffentlichen Verkehrsmitteln resultiert dabei aus der Bildung des Mittelwerts über drei unterschiedliche Aussagen (Cronbach's alpha=0,70). Die Pkw-Einstellung beruht auf einem Item (,Ich kann meinen Alltag sehr gut ohne Auto gestalten"), das umgepolt wurde. Die Einstellungen zum Fahrrad und Zufußgehen beruhen ebenfalls auf je einem Item (,Das Fahrrad ist mein alltägliches Fortbewegungsmittel“ und „Ich gehe möglichst oft zu Fuß“).

Zwischen den Einstellungen zu unterschiedlichen Verkehrsmitteln bestehen nur moderate Korrelationen $(|r|<0,5)$, sodass kein problematisches Ausmaß an Multikollinearität vorliegt (vgl. Schendera 2008: 223). Neben Verkehrsmitteleinstellungen wird die Offenheit gegenüber globaler Mobilität durch den Mittelwert aus zwei Items abgebildet. Diese lauten: „Um etwas Besonderes erzählen zu können, sollte man schon Urlaub auf einem anderen Kontinent machen“ und „Ich würde gerne mal eine Zeit lang im Ausland leben“. Die interne Reliabilität dieser Skala ist gering (Cronbach's Alpha $=0,36$ ). Wir halten es aber aus inhaltlichen Gründen für wichtig, diese Dimension abzubilden, weil bekannt ist, dass urbanes Wohnen mit großräumiger Mobilität korrespondiert (Holz-Rau/Scheiner/Sicks 2014; Czepkiewicz/ Heinonen/Ottelin 2018).

Die Verkehrsmittelnutzung wird anhand des häufigsten Verkehrsmittels für den aktuellen Bildungsweg abgebildet, also den Weg zur Hochschule, der für die Studierenden das ,Herzstück' des Alltags bildet. Sofern keine Angabe zum aktuellen Weg vorliegt, wird das jeweils zuletzt genutzte Verkehrsmittel auf Bildungswegen verwendet. Die
Aussagen werden in vier unterschiedliche Kategorien zusammengefasst: (1) zu Fuß oder Fahrrad, (2) motorisierter Individualverkehr (MIV), (3) ÖPNV und (4) wechselndes Verkehrsmittel.

Der Fragebogen lässt vielfältige Aussagen über Wohnbiografien zu, etwa die jeweiligen Orte, Gebäudetypen, Eigentumsverhältnisse, Wohndauer. Für die Analyse werden gezielt Informationen herangezogen, die bezüglich der vermuteten Einflüsse einen Beitrag leisten können. Es werden vor allem diejenigen Wohnepisoden als bedeutsam angesehen, an denen eine Person längere Zeit gewohnt hat. Die Studierenden werden einer, Ortsgrößenklasse der Kindheit und Jugend' zugeordnet, sofern sie ab einem Alter von fünf Jahren mindestens zwölf Jahre in der entsprechenden Klasse wohnhaft waren (gegebenenfalls auch an verschiedenen Orten einer Größenklasse). War eine der studierenden, befragten Personen noch in keiner Ortsgrößenklasse mindestens zwölf Jahre wohnhaft, erhält sie den Code „,wechselnd“. Die ermittelten Ortsgrößenklassen werden in Anlehnung an die Ausprägungen der Zielvariablen benannt. Ortsgrößen bis 20.000 Einwohner werden vereinfacht als „Kleinstadt/Dorf“ zusammengefasst (eine niedrigere Größenschwelle ist für die Analyse wegen geringer Fallzahlen nicht zielführend), Ortsgrößen zwischen 20.000 und 100.000 Einwohner als Mittelstadt, Ortsgrößen über 100.000 Einwohner als Großstadt.

Auch ein „Gebäudetyp der Kindheit und Jugend“ wird ermittelt (Mehrfamilienhaus vs. Einfamilien-/Doppel-/ Reihenhaus). Aufgrund fehlender Signifikanz wird die Variable im weiteren Prozess aus der Modellierung ausgeschlossen.

Bezüglich der Wohnstatuserfahrungen im bisherigen Leben (Eigentum/Miete) wird wiederum das gleiche Kriterium der Wohndauer (zwölf Jahre) herangezogen. Falls diese Dauer nicht erreicht wird, erhält diese Person den Code „wechselnd“.

Die Lage im Ort wurde auf einer fünfstufigen Likert-Skala von Ortsmitte bis Ortsrand erhoben. Daraus wird - abweichend von den obigen kategorialen Variablen, bei denen dies nicht möglich ist - näherungsweise ein Mittelwert aus den bisherigen Wohnorten gebildet. Die Lage im Ort wird somit als metrische Kovariate behandelt.

Soziodemographische Merkmale der Studierenden werden nicht verwendet. Die Altersgruppe ist äußerst homogen, und das Geschlecht zeigte bei vorläufigen Analysen keine Signifikanz.

\subsubsection{Merkmale der Eltern}

Die Verkehrsmitteleinstellung der Eltern wird ebenso ermittelt. Dabei wird die Pkw-Einstellung über den Mittelwert beider Elternteile abgebildet. Liegt ausschließlich die Einstellung der Mutter oder des Vaters vor, so wird diese als 
Pkw-Einstellung verwendet. Zusätzlich wird die Häufigkeit von Hol- und Bringdiensten der Eltern für die Studierenden während deren Kindheit als Indikator einer autoorientierten Sozialisation verwendet. Sie wird als Mittelwert aus zwei korrespondierenden Informationen gewonnen (Cronbach's Alpha $=0,735$ ), nämlich der Angabe der Eltern auf der einen (,Ich habe meine Kinder häufig mit dem Auto gefahren“) und der Studierenden auf der anderen Seite (,Meine Eltern haben mich in meiner Kindheit häufig mit dem Auto gefahren“). Dabei wird die Angabe „hatten kein Auto“ analog der Aussage „keine bzw. seltene Hol- und Bringdienste“ behandelt. Die Variable der autoorientierten Sozialisation wird letztlich aufgrund fehlender Signifikanz aus der Modellierung ausgeschlossen.

Die Wohnumfeldwünsche der Eltern sind in Bezug auf intergenerationale Zusammenhänge von besonderem Interesse. Die Codierung erfolgt analog zur Zielvariablen, mit einer Ausnahme: Die Gruppe „Antiurban“ wird nicht berücksichtigt, da urbane Wohnumfeldwünsche der Elterngeneration während der eigenen Lebensphasen als junge Erwachsene nicht feststellbar sind und somit keine eindeutig antiurbane Einstellung ausgemacht werden kann.

Der Bildungsabschluss der Elterngeneration wird als Indikator des sozialen Status berücksichtigt (Einkommensinformation liegt nicht vor). Unterschieden werden drei Kategorien: beide Eltern ohne allgemeine Hochschulreife, mindestens ein Elternteil hat allgemeine Hochschulreife und mindestens ein Elternteil hat einen Hochschulabschluss.

\subsection{Analyseverfahren}

Die Einflussfaktoren einer Variablen werden typischerweise simultan in Regressionsanalysen modelliert. Da es sich bei den Wohnumfeldwünschen um eine nominalskalierte Variable mit mehreren Ausprägungen handelt, bietet sich dazu ein multinomiales logistisches Regressionsmodell an. Dabei werden hier die genannten fünf Ausprägungen unterschieden. Jede/r Studierende gehört zu genau einer Gruppe. Die Gruppe „Suburban/Kleinstädtisch“ ist am größten und dient als Referenzkategorie. Sie ist deshalb nicht in der Ergebnistabelle (Tabelle 4) aufgeführt. Die Koeffizienten stellen die Effekte der berücksichtigten Variablen dar. Sie sind jeweils in Bezug auf die Referenzkategorie ,Suburban/Kleinstädtisch“" zu interpretieren. Dabei sind die Odds Ratios $(\operatorname{Exp}(B))$ näherungsweise als Effektstärken interpretierbar. Je weiter ein Wert von eins entfernt ist, desto stärker der Zusammenhang. Negative Zusammenhänge erscheinen in den Odds Ratios als Werte kleiner 1, positive Zusammenhänge als Werte größer 1 . An einem Beispiel verdeutlicht: Wenn ein Elternteil einen Hochschulabschluss besitzt, steigt die Wahrscheinlichkeit des studierenden Nachwuchses zur Gruppe „Urban“ zu gehören gegenüber der Referenzkategorie „Suburban/Kleinstädtisch“ auf das 4,9-fache $(\operatorname{Exp}(B)=4,91)$.

Zur Entwicklung des Regressionsmodells werden die oben beschriebenen Einflussvariablen auf Signifikanz geprüft. Im Einklang mit Backhaus/Erichson/Plinke et al. (2018: 114) wird das Regressionsmodell dem Forschungsstand folgend zunächst unter Einschluss aller unabhängigen Variablen berechnet. Erst anschließend werden einige Variablen aufgrund fehlender Signifikanz $(p>0,2)$ ausgeschlossen und das resultierende Modell auf starke Abweichungen vom ursprünglichen Modell geprüft. Dabei zeigen sich keine gravierenden Abweichungen.

\section{Ergebnisse}

\subsection{Ausprägungen der Zielvariablen}

Als Beitrag zur Reurbanisierungsdebatte ist die Verteilung der unterschiedlichen Wohnumfeldwünsche bereits für sich allein von Interesse. Tabelle 2 nennt die Prozentanteile der genannten Wünsche bezogen auf die Studierenden, die für den jeweiligen Zeithorizont eine gültige Angabe gemacht haben (Spaltenprozent, Mehrfachnennungen möglich). So möchten mehr als die Hälfte $(57,7 \%)$ der Befragten zum Befragungszeitpunkt in der Innenstadt einer Großstadt wohnen, während nur 4\% denken, diesen Wunsch auch längerfristig zu verfolgen. Umgekehrt möchten nur $19 \%$ zum Be-

Tabelle 2 Angaben der Studierenden zu ihren kurz-, mittel- und langfristigen Wohnumfeldwünschen

\begin{tabular}{llllll}
\hline & \multicolumn{2}{l}{ Wie möchten Sie wohnen? } & & & \\
& \multicolumn{2}{l}{ Anteil der Nennungen (in Prozent) } & & & \\
& jetzt & in 5-10 Jahren & längerfristig & nie & weiß nicht \\
In der Innenstadt einer Großstadt & 57,7 & 25,8 & 4,0 & 35,4 & 20,4 \\
Am Stadtrand einer Großstadt & 23,1 & 36,6 & 29,0 & 11,1 & 36,7 \\
In einer kleinen oder mittelgroßen Stadt & 14,1 & 26,5 & 30,6 & 18,7 & 44,4 \\
Auf dem Land/Dorf & 4,8 & 7,3 & 18,9 & 64,9 & 39,3 \\
In einem Haus/einer Wohnung mit Garten & 16,3 & 26,8 & 64,6 & 2,5 & 15,0 \\
\hline
\end{tabular}

Angaben in Spaltenprozent derjenigen, die für den jeweiligen Zeithorizont eine gültige Angabe gemacht haben. Mehrfachnennungen möglich. 
Tabelle 3 Zielvariable Wohnumfeldwünsche

\begin{tabular}{lll}
\hline Ausprägung Wohnumfeldwunsch & Fälle & Prozent \\
Urban & 29 & 5,3 \\
Antiurban & 155 & 28,1 \\
Suburban/Kleinstädtisch & 262 & 47,5 \\
Ländlich & 52 & 9,4 \\
Offen & 54 & 9,8 \\
Summe & 552 & 100,0 \\
\hline
\end{tabular}

fragungszeitpunkt in einer kleineren Stadt (14\%) oder auf dem Land $(5 \%)$ wohnen; längerfristig trifft dies aber für die Hälfte der Studierenden zu (30,6+18,9=49,5\%).

Die fünf Ausprägungen der Zielvariablen zeigen ebenfalls ein eindeutiges Bild. Die Gruppe „Urban“ stellt mit 29 Studierenden die kleinste Gruppe dar (vgl. Tabelle 3), obwohl diese Befragten teils sogar noch weitere Wünsche geäußert haben. Der urbane Wohnumfeldwunsch scheint nur während des Studiums sehr präsent, längerfristig zeigt sich eine deutliche Tendenz Richtung Stadtrand und Kleinoder Mittelstadt. Mit 262 Fällen ist die Gruppe „Suburban/ Kleinstädtisch“ am stärksten vertreten. Insgesamt 155 Studierende sind der Gruppe „Antiurban“ zuzuordnen, welche einen urbanen Wohnumfeldwunsch sowohl jetzt als auch in zukünftigen Lebensphasen komplett ausschließen. Des Weiteren präferieren 52 Studierende längerfristig einen ländlichen Wohnstandort, während 54 eine noch offene Perspektive angeben.

\subsection{Modellschätzungen der Regressionsanalyse}

Die Tabellen 4 und 5 zeigen die deskriptiven Verteilungen der verwendeten Variablen. Aus inhaltlicher Sicht von Interesse sind die Verkehrsmitteleinstellungen der Studierenden - die Zustimmung zum Pkw fällt deutlich stärker aus als die Zustimmung zum ÖPNV oder zum Gehen (vgl. Tabelle 5). Dies stützt nicht die in der Forschung behauptete Pkw-Aversion junger Erwachsener. Die Pkw-Einstellung der Elterngeneration ist allerdings noch positiver geprägt. Dies korrespondiert mit den suburban bis ländlich gepräg-
Tabelle 4 Nominale Variablen in der Regressionsanalyse

\begin{tabular}{|c|c|c|c|}
\hline \multicolumn{2}{|l|}{ Variablen } & Anzahl & Prozent \\
\hline \multirow{4}{*}{$\begin{array}{l}\text { Hauptverkehrs- } \\
\text { mittel des } \\
\text { aktuellen } \\
\text { (alternativ: des } \\
\text { letzten) }\end{array}$} & Wechselnd & 31 & 6 \\
\hline & Zu Fuß oder Fahrrad & 97 & 18 \\
\hline & MIV & 80 & 14 \\
\hline & ÖPNV & 344 & 62 \\
\hline \multicolumn{4}{|l|}{ Bildungswegs } \\
\hline \multirow{4}{*}{$\begin{array}{l}\text { Jetziger Wohn- } \\
\text { umfeldwunsch } \\
\text { der Eltern }\end{array}$} & Unklar & 65 & 12 \\
\hline & $\begin{array}{l}\text { Innenstadt einer } \\
\text { Großstadt }\end{array}$ & 44 & 8 \\
\hline & Land/Dorf & 127 & 23 \\
\hline & $\begin{array}{l}\text { Stadtrand oder Klein-/ } \\
\text { Mittelstadt }\end{array}$ & 316 & 57 \\
\hline \multirow{3}{*}{$\begin{array}{l}\text { Höchster Bil- } \\
\text { dungsabschluss } \\
\text { mindestens } \\
\text { eines Elternteils }\end{array}$} & Hochschulabschluss & 275 & 50 \\
\hline & $\begin{array}{l}\text { Mit allgemeiner Hoch- } \\
\text { schulreife }\end{array}$ & 143 & 26 \\
\hline & $\begin{array}{l}\text { Ohne allgemeine } \\
\text { Hochschulreife }\end{array}$ & 134 & 24 \\
\hline \multirow{4}{*}{$\begin{array}{l}\text { Prägende Orts- } \\
\text { größenklasse in } \\
\text { der Kindheit } \\
\text { und Jugend (ab } \\
\text { einem Alter von } \\
\text { fünf Jahren) }\end{array}$} & Wechselnd & 31 & 6 \\
\hline & Großstadt & 210 & 38 \\
\hline & Kleinstadt/Dorf & 151 & 27 \\
\hline & Mittelstadt & 160 & 29 \\
\hline \multirow{3}{*}{$\begin{array}{l}\text { Wohnstatus in } \\
\text { der Kindheit } \\
\text { und Jugend }\end{array}$} & Wechselnd & 75 & 14 \\
\hline & Miete & 135 & 24 \\
\hline & Eigentum & 342 & 62 \\
\hline
\end{tabular}

ten Wohnumfeldwünschen der Eltern (die häufig mit deren tatsächlichen Wohnorten zusammenfallen).

Es wurden verschiedene wohnbiographische Variablen geprüft: die Ortsgröße, die Lage im Gesamtort, der Gebäudetyp und der Wohnstatus (Miete oder Eigentum). Dabei zeigen sich folgende signifikante Zusammenhänge (vgl. Tabelle 6): Erstens erhöht eine ländlich geprägte Ortsgrößenklasse in der Kindheit und Jugend die Wahrscheinlichkeit für die Gruppe „Ländlich“ relativ zur Referenzkategorie „Suburban/Kleinstädtisch“ um den Faktor 3,232. Zweitens zeigt sich ein Einfluss des Wohnstatus. Bei Studierenden, die mit wechselndem Wohnstatus aufgewachsen sind (Wechsel zwischen Miete und Eigentum), erhöht sich die Wahrscheinlichkeit, der Gruppe „Urban“

Tabelle 5 Metrische Variablen in der Regressionsanalyse

\begin{tabular}{lll}
\hline Variablen & Mittelwert & Standardabweichung \\
ÖPNV-Einstellung (1 (Abneigung) bis 5 (Zuneigung)) & 2,36 & 0,92 \\
Pkw-Einstellung (1 (Abneigung) bis 5 (Zuneigung)) & 3,78 & 1,20 \\
Zu Fuß-Einstellung (1 (Abneigung) bis 5 (Zuneigung)) & 2,77 & 1,11 \\
Globale Mobilität (1 (Abneigung) bis 5 (Zuneigung)) & 2,73 & 0,83 \\
Eltern: Pkw-Einstellung (1 (Abneigung) bis 5 (Zuneigung)) & 4,27 & 0,90 \\
Lage der prägenden Wohnsituation (ab einem Alter von fünf Jahren) im Gesamtort & 3,20 & 1,25 \\
(1 (Ortsmitte) bis 5 (Ortsrand)) & & \\
\hline
\end{tabular}


anzugehören, um das Vierfache $(\operatorname{Exp}(B)=4,053)$. Auch das Aufwachsen in einer Mietwohnung ist mit einer höheren Wahrscheinlichkeit mit urbanen Wohnumfeldwünschen verbunden $(\operatorname{Exp}(B)=2,792)$. „Bewegte“ Wohnbiographien (Wechsel des Wohnstatus) und das Aufwachsen in Mietwohnungen ist demnach mit einer stärkeren urbanen Neigung verbunden. Die kleinräumliche Lage im Ort zeigt keine signifikanten Zusammenhänge. Alles in allem deutet dies auf mögliche langfristige Prägungen von Wohnumfeldwünschen hin. Für die Reurbanisierungsdebatte bedeutet dies, dass die zunehmende Sozialisation im Eigentum und/oder in suburbanen Umfeldern entsprechende Wünsche für die Zukunft stärkt.

Zur Untersuchung intergenerationaler Effekte wurde eine Reihe an Einflussvariablen herangezogen. Die Wünsche der Eltern (zum Zeitpunkt der Befragung) stechen dabei besonders heraus. Ein urbaner Wohnumfeldwunsch der Eltern führt zu einer enorm höheren Wahrscheinlichkeit ihres erwachsenen Kindes, ebenfalls urban wohnen zu wollen $(\operatorname{Exp}(B)=10,269)$. Analog zeigt sich ein starker Einfluss ländlicher Wohnumfeldwünsche der Eltern auf die ländlichen Wohnumfeldwünsche der Studierenden $(\operatorname{Exp}(B)=2,966)$. Dazu passend deutet sich ein positiver Effekt des ländlichen Wohnumfeldwunsches der Eltern auf die Zugehörigkeit zur Gruppe „Antiurban“ an $(\operatorname{Exp}(B)=1,681$, allerdings insignifikant, $\mathrm{p}=0,104)$. Dies spricht wiederum für eine Selbstverstärkung von Raumentwicklungen durch Sozialisation, die einer Reurbanisierung als Megatrend möglicherweise entgegenstehen.

Allerdings findet sich auch ein Zusammenhang, der gegen eine solche Interpretation spricht. Eine positive Pkw-Einstellung der Eltern erhöht die Wahrscheinlichkeit für ihr Kind - der/die befragte Studierende -, längerfristig in der Innenstadt einer Großstadt wohnen zu wollen $(\operatorname{Exp}(B)=1,959)$. Dieses Ergebnis widerspricht der Vermutung, dass umgekehrt eine Pkw-Abneigung der Eltern zu urbanen Wohnumfeldwünschen der Kinder führt. Möglicherweise deutet sich hier in Ergänzung der bekannten Pkw-Aversion junger Erwachsener eine Art Protesthaltung gerade derjenigen an, die mit starker Pkw-Orientierung aufgewachsen sind. Weitere Verkehrsmitteleinstellungen der Eltern waren nicht signifikant, sodass diese bereits im Vorfeld aus dem Modell ausgeschlossen wurden.

Signifikante Zusammenhänge sind außerdem zwischen dem Bildungsabschluss der Eltern und den Wünschen der Studierenden festzustellen. Ein hoher Bildungsstatus der Eltern ist mit urbanen oder offenen Wohnumfeldwünschen ihrer Kinder assoziiert. Für Elternteile mit Hochschulabschluss betragen die Werte $\operatorname{Exp}(B)=4,912$ für einen urbanen Wohnumfeldwunsch und $\operatorname{Exp}(B)=2,772$ für einen offenen Wohnumfeldwunsch. Für Elternteile mit Abitur (gegenüber solchen ohne Abitur) liegen die Werte in einer ähnlichen
Größenordnung. In Ergänzung der bekannten höheren Bildungsniveaus der Bevölkerung in Städten zeigt sich hier eine entsprechende, intergenerationale Transmission".

Die Effekte der Verkehrsmitteleinstellungen der befragten Studierenden bestätigen die Erwartungen. Im Vergleich zur Referenzgruppe „Suburban/Kleinstädtisch“ steigt die Wahrscheinlichkeit der Zugehörigkeit zur Gruppe „Urban“ mit jeder Zunahme einer positiven Einstellung gegenüber dem ÖPNV um eine Einheit auf etwa das Doppelte $(\operatorname{Exp}(B)=1,996)$. Eine positive Einstellung gegenüber den öffentlichen Verkehrsmitteln führt also zu einer höheren Wahrscheinlichkeit, zukünftig urban wohnen zu wollen. Demgegenüber sinkt die Wahrscheinlichkeit, der Gruppe „Antiurban“ anzugehören, um etwa ein Drittel $(\operatorname{Exp}(B)=0,695)$, wenn die ÖPNV-Zuneigung sich um eine Einheit erhöht.

Umgekehrt erhöht eine stärkere Neigung zum Pkw die Wahrscheinlichkeit, niemals in der Innenstadt einer Großstadt wohnen zu wollen $(\operatorname{Exp}(B)=1,254)$. Bezüglich des $\mathrm{Zu}-$ fußgehens zeigt sich kein signifikanter Zusammenhang. Offenbleiben muss an dieser Stelle die Entstehung differenzierter Einstellungen zu Verkehrsmitteln. Sozialisatorische Effekte sind auch hier zu vermuten. Wichtig ist es in jedem Fall festzuhalten, dass das Potenzial für künftige Reurbanisierung mit entsprechenden Präferenzen für den ÖPNV bzw. das Auto korrespondiert.

Zusätzlich untersuchten wir die Offenheit gegenüber globaler Mobilität. Es zeigte sich, dass Befragte, die globale Mobilität positiv bewerten, mit geringerer Wahrscheinlichkeit antiurban eingestellt sind $(\operatorname{Exp}(B)=0,565)$ und auch mit geringerer Wahrscheinlichkeit zukünftig ländlich leben möchten $(\operatorname{Exp}(B)=0,646)$. Insgesamt trifft dies nicht punktgenau, aber bestätigt tendenziell die vorliegenden Befunde der Verkehrsforschung, dass die Bevölkerung von größeren Städten bzw. Verdichtungsräumen in ihrer Mobilität großräumiger orientiert ist als die ländliche Bevölkerung (HolzRau/Scheiner/Sicks 2014; Czepkiewicz/Heinonen/Ottelin 2018).

Sofern die Studierenden den MIV nutzen, steigt die Wahrscheinlichkeit, der Kategorie „Ländlich“ anzugehören, stark an $(\operatorname{Exp}(B)=3,810)$. Wird der Bildungsweg zu Fuß oder mit dem Fahrrad zurückgelegt, deutet sich ebenfalls ein positiver Einfluss $(\operatorname{Exp}(B)=2,169)$ auf einen ländlichen Wohnumfeldwunsch an. Dieser verfehlt allerdings knapp das Signifikanzniveau ( $\mathrm{p}=0,066)$.

Des Weiteren zeigen sich für die Gruppe „Offen“ nennenswerte Ergebnisse. Die Wahrscheinlichkeit eines noch offenen Wohnumfeldwunsches steigt mit dem Hauptverkehrsmittel „,zu Fuß“ oder „Fahrrad“ an $(\operatorname{Exp}(B)=2,763)$. Mögliche Gründe müssen an dieser Stelle offenbleiben. Wechselnde Hauptverkehrsmittel sind ebenfalls mit einer höheren Wahrscheinlichkeit $(\operatorname{Exp}(B)=2,798)$ für einen 
Tabelle 6 Modellschätzungen der multinomialen logistischen Regressionsanalyse

\begin{tabular}{|c|c|c|c|c|c|c|c|c|c|c|c|c|c|}
\hline & & \multicolumn{3}{|l|}{ Urban } & \multicolumn{3}{|c|}{ Antiurban } & \multicolumn{3}{|c|}{ Ländlich } & \multicolumn{3}{|l|}{ Offen } \\
\hline \multirow{2}{*}{ Konstante } & \multirow[b]{3}{*}{$\begin{array}{l}1 \text { (Abneigung) bis } \\
5 \text { (Zuneigung) }\end{array}$} & \multirow{2}{*}{$\begin{array}{l}B \\
-0,658\end{array}$} & \multicolumn{2}{|l|}{$\operatorname{Exp}(B)$} & \multirow{2}{*}{$\begin{array}{l}B \\
-2,257\end{array}$} & \multicolumn{2}{|l|}{$\operatorname{Exp}(B)$} & \multirow{2}{*}{$\begin{array}{l}B \\
-5,048\end{array}$} & \multicolumn{2}{|l|}{$\operatorname{Exp}(B)$} & \multirow{2}{*}{$\begin{array}{l}B \\
-1,663\end{array}$} & \multicolumn{2}{|l|}{$\operatorname{Exp}(B)$} \\
\hline & & & & & & & & & & & & & \\
\hline ÖPNV-Einstellung & & 0,691 & 1,996 & ** & $-0,364$ & 0,695 & $* * *$ & 0,083 & 1,087 & & $-0,087$ & 0,917 & \\
\hline Pkw-Einstellung & $\begin{array}{l}1 \text { (Abneigung) bis } \\
5 \text { (Zuneigung) }\end{array}$ & 0,150 & 1,162 & & 0,226 & 1,254 & ** & $-0,094$ & 0,911 & & 0,065 & 1,067 & \\
\hline Zu Fuß-Einstellung & $\begin{array}{l}1 \text { (Abneigung) bis } \\
5 \text { (Zuneigung) }\end{array}$ & $-0,331$ & 0,718 & * & 0,164 & 1,178 & * & 0,067 & 1,070 & & 0,188 & 1,207 & \\
\hline \multirow{3}{*}{$\begin{array}{l}\text { Hauptverkehrsmittel } \\
\text { des aktuellen } \\
\text { (alternativ: des } \\
\text { letzten) } \\
\text { Bildungsweges }\end{array}$} & Wechselnd & $-0,489$ & 0,613 & & 0,043 & 1,043 & & - & - & - & 1,029 & 2,798 & * \\
\hline & $\begin{array}{l}\text { Zu Fuß oder Fahr- } \\
\text { rad }\end{array}$ & 0,430 & 1,537 & & 0,146 & 1,157 & & 0,774 & 2,169 & * & 1,016 & 2,763 & $\star * *$ \\
\hline & $\begin{array}{l}\text { MIV } \\
\text { ÖPNV (Referenz) }\end{array}$ & 0,016 & 1,016 & & 0,281 & 1,324 & & 1,338 & 3,810 & $* * *$ & 0,540 & 1,715 & \\
\hline Globale Mobilität & $\begin{array}{l}1 \text { (nicht offen) bis } \\
5 \text { (offen) }\end{array}$ & $-0,186$ & 0,831 & & $-0,571$ & 0,565 & $* * *$ & $-0,438$ & 0,646 & ** & $-0,139$ & 0,870 & \\
\hline $\begin{array}{l}\text { Eltern: Pkw-Einstel- } \\
\text { lung }\end{array}$ & $\begin{array}{l}1 \text { (Abneigung) bis } \\
5 \text { (Zuneigung) }\end{array}$ & 0,672 & 1,959 & $* * *$ & 0,101 & 1,106 & & $-0,148$ & 0,863 & & 0,121 & 1,129 & \\
\hline \multirow{4}{*}{$\begin{array}{l}\text { Jetziger Wohnum- } \\
\text { feldwunsch der } \\
\text { Eltern }\end{array}$} & Unklar & $-0,041$ & 0,960 & & $-0,310$ & 0,734 & & 0,025 & 1,025 & & 0,166 & 1,181 & \\
\hline & $\begin{array}{l}\text { Innenstadt einer } \\
\text { Großstadt }\end{array}$ & 2,329 & 10,269 & $* * *$ & $-0,726$ & 0,484 & & 0,200 & 1,221 & & 0,509 & 1,664 & \\
\hline & Land/Dorf & $-0,039$ & 0,962 & & 0,520 & 1,681 & * & 1,087 & 2,966 & $* *$ & 0,541 & 1,717 & \\
\hline & $\begin{array}{l}\text { Stadtrand } \\
\text { oder Klein-/ } \\
\text { Mittelstadt (Refe- } \\
\text { renz) }\end{array}$ & & & & & & & & & & & & \\
\hline \multirow{3}{*}{$\begin{array}{l}\text { Höchster } \\
\text { Bildungsabschluss } \\
\text { mindestens eines } \\
\text { Elternteils }\end{array}$} & $\begin{array}{l}\text { Hochschul- } \\
\text { abschluss }\end{array}$ & 1,592 & 4,912 & $* *$ & $-0,087$ & 0,916 & & 0,049 & 1,051 & & 1,020 & 2,772 & $* *$ \\
\hline & $\begin{array}{l}\text { Mit allgemeiner } \\
\text { Hochschulreife }\end{array}$ & 1,327 & 3,769 & * & 0,463 & 1,589 & * & $-0,071$ & 0,931 & & 1,073 & 2,925 & $\star *$ \\
\hline & $\begin{array}{l}\text { Ohne allgemeine } \\
\text { Hochschulreife } \\
\text { (Referenz) }\end{array}$ & & & & & & & & & & & & \\
\hline \multirow{4}{*}{$\begin{array}{l}\text { Prägende } \\
\text { Ortsgrößenklasse } \\
\text { in der Kindheit und } \\
\text { Jugend (ab einem } \\
\text { Alter von } 5 \text { Jahren) }\end{array}$} & Wechselnd & $-0,170$ & 0,844 & & $-0,203$ & 0,816 & & $-0,826$ & 0,438 & & 0,131 & 1,140 & \\
\hline & Großstadt & 0,095 & 1,099 & & $-0,219$ & 0,803 & & $-0,245$ & 0,782 & & 0,073 & 1,076 & \\
\hline & Kleinstadt/Dorf & 0,999 & 2,716 & * & 0,158 & 1,171 & & 1,173 & 3,232 & $* *$ & 0,172 & 1,188 & \\
\hline & $\begin{array}{l}\text { Mittelstadt (Refe- } \\
\text { renz) }\end{array}$ & & & & & & & & & & & & \\
\hline \multirow{3}{*}{$\begin{array}{l}\text { Wohnstatus in der } \\
\text { Kindheit und } \\
\text { Jugend }\end{array}$} & Wechselnd & 1,399 & 4,053 & $* *$ & $-0,221$ & 0,802 & & 0,337 & 1,401 & & 0,321 & 1,378 & \\
\hline & Miete & 1,027 & 2,792 & * & 0,195 & 1,215 & & $-0,213$ & 0,808 & & 0,528 & 1,696 & * \\
\hline & $\begin{array}{l}\text { Eigentum (Refe- } \\
\text { renz) }\end{array}$ & & & & & & & & & & & & \\
\hline $\begin{array}{l}\text { Lage der prägenden } \\
\text { Wohnsituation } \\
\text { (ab einem Alter } \\
\text { von } 5 \text { Jahren) im } \\
\text { Gesamtort }\end{array}$ & $\begin{array}{l}1 \text { (Ortsmitte) bis } \\
5 \text { (Ortsrand) }\end{array}$ & 0,298 & 1,347 & * & 0,153 & 1,166 & * & 0,116 & 1,122 & & 0,081 & 1,084 & \\
\hline Cox und Snell & 0,301 & Nagelke & erke & & 0,324 & & & McFadc & len & & 0,136 & & \\
\hline
\end{tabular}

*** hochsignifikant $(p<0,01) / * *$ signifikant $(p<0,05) /$ * gerade insignifikant $(p<0,20)$ 
noch offenen Wohnumfeldwunsch assoziiert (allerdings knapp insignifikant). Dies legt nahe, dass Multimodalität möglicherweise in einem weiter verstandenen Sinne eine Offenheit zum Ausdruck bringt, die sich auch bezüglich künftiger Wünsche niederschlägt.

\section{Fazit und Schlussfolgerungen}

Die Verteilung der mittel- und langfristigen Wohnumfeldwünsche der Studierenden zeigt, dass strikt urbane Wohnumfeldwünsche keine bedeutende Rolle in der Reurbanisierungsdebatte spielen. Langfristig dominieren vielmehr die Kategorien „Suburban/Kleinstädtisch“ und sogar „Antiurban“. Somit geht die große Mehrzahl der jungen Erwachsenen zu Beginn ihres Studiums davon aus, dass städtisch geprägte Wohnstandorte für sie allenfalls eine Durchgangsstation darstellen. Damit werden durch unsere Befragung unter Studierenden der Raumplanung der TU Dortmund Ergebnisse bestätigt und konkretisiert, die für Deutschland aus kleineren experimentellen Umfragen vorliegen (von Einem 2016; Weller 2016).

Bei den vorgenommenen Modellschätzungen konnten wichtige Einflussgrößen auf die späteren Wohnumfeldwünsche Studierender identifiziert werden. Die empirischen Befunde bestätigen überwiegend positive Effekte intergenerationaler Sozialisation und Erfahrungen, sodass die Weitergabe von Werten, Normen oder Einstellungen der Eltern für den Wohnumfeldwunsch relevant ist. Der stärkste Einfluss zeigt sich zwischen dem urbanen Wohnumfeldwunsch der Eltern und dem urbanen Wohnumfeldwunsch der Studierenden. Auf den ersten Blick überraschend erscheint das Ergebnis, dass die Pkw-Orientierung der Eltern eine stärkere (nicht schwächere) urbane Orientierung des Kindes hervorruft. Ein möglicher Erklärungsansatz hierfür könnte in einer Art Protesthaltung der jungen Erwachsenen liegen. Von einem Einfluss der eigenen Wohnbiographie ist ebenfalls auszugehen. Eine Kindheit und Jugend in kleineren Kommunen erhöht die Wahrscheinlichkeit einer Präferenz für ländliche Wohnumfeldwünsche. Bei „bewegten“ Wohnbiographien und einem Aufwachsen in Mietwohnungen ist eine urbane Orientierung häufiger. Die auf dem Bildungsweg genutzten Hauptverkehrsmittel während des Studiums weisen auf die wichtige Bedeutung der Alltagsmobilität hin. Dies zeigt sich besonders deutlich in der Nutzung des MIV im Zusammenhang mit einem ländlichen Wohnumfeldwunsch.

Im Hinblick auf die künftige Raumentwicklung kann folgende Schlussfolgerung aus dieser Studie gezogen werden: Die Ergebnisse sprechen insgesamt deutlich dagegen, dass der in den letzten Jahren zu beobachtende Reurbanisierungstrend mit grundlegend veränderten Präferenzen einer neuen
Generation verbunden ist, die sich auch in zukünftigen Lebensphasen in einer stärkeren Orientierung zugunsten urbaner Wohnstandorte niederschlagen werden. Das Gegenteil scheint der Fall zu sein: Die Präferenzen der neuen Generation sind durch deren eigene Erfahrungen in der Kindheit und Jugend geprägt, und diese Erfahrungen sind zunehmend durch Eigentum und suburbane Umfelder bestimmt. Seit Beginn der Reurbanisierungsdebatte wurde auch immer wieder mit Vorsicht, Zurückhaltung und Skepsis über die Dauerhaftigkeit dieses Trends diskutiert (z. B. Hirschle/ Schürt 2008; Growe/Münter 2010; Hesse 2010). In der jüngeren Vergangenheit wurde aufgrund einiger Publikationen zudem ein „Ende der Reurbanisierung“ ausgerufen. Auch gab es empirische ,Belege ' für eine erneute Trendverschiebung, da aufgrund der angespannten Wohnungsmärkte in den Kernstädten Prozesse der Suburbanisierung wieder deutlich an Bedeutung gewonnen hätten (z. B. Kholodilin 2017; Osterhage 2018; Held/Mäding 2020). Über diese durch Miet- und Kaufpreise ,erzwungene' Dezentralisierung hinaus unterstreichen die vorliegenden Ergebnisse, dass auch die Wohnumfeldwünsche der Generation $\mathrm{Z}$ gegen einen Reurbanisierungstrend als Selbstläufer sprechen. Im Sinne der Charakterisierung der Generation Z (Albert/Hurrelmann/Quenzel et al. 2019) lässt sich unser zentraler Befund möglicherweise im Sinne einer Ambivalenz zwischen normativen Vorstellungen zu Umwelt- und Klimaschutz einerseits und persönlichen Lebensvorstellungen andererseits interpretieren, in denen sich ein gewisser Konservatismus (Geschlechterrollen, Familie, Häuschen im Grünen) mit Hedonismus und einer ausgewogenen WorkLife-Balance verbinden.

Für die Praxis der Stadt- und Regionalentwicklung macht dies die Arbeit nicht leichter. Kommunen und Regionen sind einer nachhaltigen Raumentwicklung verpflichtet. Hierzu gehören Klimaschutz, Reduktion der Flächeninanspruchnahme und umweltverträgliche Verkehrsentwicklung, die unter teils kontraproduktiven Rahmenbedingungen (Erhöhung der Entfernungspauschale, Baulandmobilisierungsgesetz) zu verfolgen sind. Gleichzeitig dürfte unstrittig sein, dass es in einer liberalen Demokratie grundsätzlich möglich sein sollte, individuelle Wohn- und Lebensvorstellungen zu realisieren. Für die planerische Praxis bleibt es somit eine drängende Aufgabe, gleichermaßen attraktive wie zukunftsweisende Wohnangebote zu schaffen, die einerseits individuelle Wohnwünsche und Bedürfnisse berücksichtigen sowie andererseits zentralen gesellschaftlichen Herausforderungen gerecht werden, und zwar in unterschiedlichen räumlichen Kontexten und nicht nur in den urbanen Gebieten von Großstädten. Dies erfordert begleitend entsprechende anwendungsbezogene Forschung.

Bei der Einordnung der Untersuchungsergebnisse muss allerdings selbstverständlich die Zusammensetzung der zu- 
grundeliegenden Stichprobe berücksichtigt werden. Im vorliegenden Fall wurden ausschließlich Studierende des Studiengangs Raumplanung an der Technischen Universität Dortmund befragt, die noch am Anfang ihres Studiums standen. Durch den gewählten Untersuchungsansatz war es möglich, eine komplexe Befragung nahezu als Vollerhebung durchzuführen und somit einen in dieser Form einzigartigen Datensatz zu schaffen. Für zukünftige Forschungsarbeiten sind vor diesem Hintergrund zwei Fragestellungen von besonderem Interesse: Zum einen geht es um die Frage, ob sich die vorliegenden Befunde auch für andere junge Erwachsene - Studierende anderer Fachrichtungen und NichtStudierende an anderen Standorten - bestätigen lassen. Unsere Vermutung ist, dass andere Berufsgruppen eher noch weniger urban orientiert sind, weil Urbanität in der Raumplanung aufgrund der ökologisch-sozialen Ausrichtung des Faches weitgehend positiv konnotiert ist. Zum anderen sollte der Frage nachgegangen werden, inwieweit sich die geäußerten mittel- und langfristigen Wohnumfeldwünsche im Laufe des Studiums möglicherweise noch verschieben, da im Laufe der Zeit sowohl positive als auch negative Wohnerfahrungen an einem urbanen Standort gesammelt werden.

\section{Literatur}

Aero, T. (2006): Residential Choice from a Lifestyle Perspective. In: Housing, Theory and Society 23, 2, 109-130. https://doi.org/10.1080/14036090600773139

Albert, M.; Hurrelmann, K.; Quenzel, G.; Schneekloth, U. (2019): 18. Shell Jugendstudie. Jugend 2019. Eine Generation meldet sich zu Wort. Hamburg.

Albrecht, J. (2015): Die räumliche Bindung an den Wohnort der Kindheit und Jugend in der Familiengründungsphase. Wohnstandortentscheidungen im Biografien- und Generationenansatz. In: Scheiner, J.; Holz-Rau, C. (Hrsg.): Räumliche Mobilität und Lebenslauf. Studien zu Mobilitätsbiografien und Mobilitätssozialisation. Wiesbaden, 241-260. https://doi.org/10.1007/978-3-658-07546-0_ 13

Albrecht, J.; Döring, L.; Holz-Rau, C.; Scheiner, J. (2019): Residential Location Choices of Couples Considering both Partners' Residential Biographies and Family Ties. In: Comparative Population Studies 44, 107-136. https:// doi.org/10.12765/CPoS-2019-12en

ARL - Akademie für Raumforschung und Landesplanung (2018): Reurbanisierung in nordwestdeutschen Städten und Regionen. Befunde, Handlungsempfehlungen, Forschungsbedarf. Hannover. $=$ Positionspapier aus der ARL 110.

Backhaus, K.; Erichson, B.; Plinke, W.; Weiber, R. (2018): Multivariate Analysemethoden. Eine anwendungsorien- tierte Einführung. Berlin. https://doi.org/10.1007/978-3662-56655-8

Blaauboer, M. (2011): The Impact of Childhood Experiences and Family Members Outside the Household on Residential Environment Choices. In: Urban Studies 48, 8, 1635-1650. https://doi.org/10.1177/ 0042098010377473

Brake, K.; Herfert, G. (Hrsg.) (2012): Reurbanisierung. Materialität und Diskurs in Deutschland. Wiesbaden. https://doi.org/10.1007/978-3-531-94211-7

Brake, K.; Urbanczyk, R. (2012): Reurbanisierung - Strukturierung einer begrifflichen Vielfalt. In: Brake, K.; Herfert, G. (Hrsg.): Reurbanisierung. Materialität und Diskurs in Deutschland. Wiesbaden, 34-51. https://doi.org/ 10.1007/978-3-531-94211-7_3

Brombach, K.; Jessen, J. (2017): Reurbanisierung und Wohnungsbau in Stuttgart. In: Standort - Zeitschrift für Angewandte Geographie 41, 3, 173-179. https://doi.org/10. 1007/s00548-017-0494-9

Brühl, H.; Echter, C.-P.; Frölich von Bodelschwingh, F.; Jekel, G. (2005): Wohnen in der Innenstadt - eine Renaissance ? Berlin. = Difu-Beiträge zur Stadtforschung 41.

Bucher, H.; Schlömer, C. (2012): Eine demografische Einordnung der Re-Urbanisierung. In: BBSR - Bundesinstitut für Bau-, Stadt- und Raumforschung (Hrsg.): Die Attraktivität großer Städte: ökonomisch, demografisch, kulturell. Ergebnisse eines Ressortforschungsprojekts des Bundes. Bonn, 66-72.

Busch, R. (2016): Inländische Wanderungen in Deutschland - wer gewinnt und wer verliert? In: Zeitschrift für Immobilienökonomie 2, 2, 81-101. https://doi.org/10.1365/ s41056-016-0012-3

Caulfield, J. (1994): City Form and Everyday Life: Toronto's Gentrification and Critical Social Practice. Toronto.

Coulter, R.; van Ham, M.; Findlay, A. M. (2016): Re-thinking residential mobility: Linking lives through time and space. In: Progress in Human Geography 40, 3, 352-374. https://doi.org/10.1177/0309132515575417

Czepkiewicz, M.; Heinonen, J.; Ottelin, J. (2018): Why do urbanites travel more than do others? A review of associations between urban form and long-distance leisure travel. In: Environmental Research Letters 13, 7, 073001. https://doi.org/10.1088/1748-9326/aac9d2

Delbosc, A.; Naznin, F. (2019): Future Life Course and Mobility: A Latent Class Analysis of Young Adults in Victoria, Australia. In: Transport Policy 77, 104-116. https://doi.org/10.1016/j.tranpol.2019.03.005

Dembski, S.; Sykes, O.; Couch, C.; Desjardins, X.; Evers, D.; Osterhage, F.; Siedentop, S.; Zimmermann, K. (2019): Reurbanisation and suburbia in Northwest Europe: A comparative perspective on spatial trends and 
policy approaches. In: Progress in Planning. https://doi. org/10.1016/j.progress.2019.100462

Dittrich-Wesbuer, A.; Osterhage, F. (2008): Wohnstandortentscheidungen in der Stadtregion: das Beispiel „Bergisches Land“. Dortmund. = ILS-Trends 2/2008.

Döring, L. (2015): Biografieeffekte und intergenerationale Sozialisationseffekte in Mobilitätsbiografien. In: Scheiner, J.; Holz-Rau, C. (Hrsg.): Räumliche Mobilität und Lebenslauf. Studien zu Mobilitätsbiografien und Mobilitätssozialisation. Wiesbaden, 23-41. https://doi.org/10. 1007/978-3-658-07546-0_2

Döring, L.; Albrecht, J.; Scheiner, J.; Holz-Rau, C. (2014): Mobility Biographies in Three Generations - Socialization Effects on Commute Mode Choice. In: Transportation Research Procedia 1, 1, 165-176. https://doi.org/10. 1016/j.trpro.2014.07.017

Engler, P. (2014): Reurbanisierung und Wohnwünsche: Die Bedeutung städtischer Strukturen für die Bevölkerung in der Stadtregion Hamburg. Münster. = Schriften des Arbeitskreises Stadtzukünfte der Deutschen Gesellschaft für Geographie 13.

Feijten, P.; Hooimeijer, P.; Mulder, C. H. (2008): Residential Experience and Residential Environment Choice over the Life-course. In: Urban Studies 45, 1, 141-162. https:// doi.org/10.1177/0042098007085105

Findlay, A.; McCollum, D.; Coulter, R.; Gayle, V. (2015): New Mobilities Across the Life Course: a Framework for Analysing Demographically Linked Drivers of Migration. In: Population, Space and Place 21, 4, 390-402. https://doi.org/10.1002/psp.1956

Frank, S. (2018): Inner-City Suburbanization - no Contradiction in Terms. Middle-Class Family Enclaves are Spreading in the Cities. In: Raumforschung und Raumordnung | Spatial Research and Planning 76, 2, 123-132. https://doi.org/10.1007/s13147-016-0444-1

Fuchs, M.; Nadler, R.; Roth, D.; Theuer, S.; Weyh, A. (2017): Rückwanderung von Erwerbspersonen - aktuelle Deutschlandzahlen im regionalen Vergleich. http:// aktuell.nationalatlas.de/rueckwanderung-4_05-2017-0$\mathrm{html} /$ (23.06.2021).

Gebert, S. (2017): Dorfbewohner kämpfen gegen Landflucht. https://www.deutschlandfunk.de/reurbanisierungdorfbewohner-kaempfen-gegen-landflucht.1775.de.html? dram:article_id=401752 (23.06.2021).

Gerber, K. (2011): Räumliche Mobilität im Wandel. Wanderungen im Lebenslauf und ihre Auswirkungen auf die Stadtentwicklung in Nordrhein-Westfalen. Wiesbaden. https://doi.org/10.1007/978-3-531-92909-5

Glatter, J.; Hackenberg, K.; Wolff, M. (2014): Zimmer frei? Die Wiederentdeckung der Relevanz des studentischen Wohnens für lokale Wohnungsmärkte. In: Raumfor- schung und Raumordnung 72, 5, 385 - 399. https://doi. org/10.1007/s13147-014-0303-x

Growe, A.; Münter, A. (2010): Die Renaissance der großen Städte. In: Geographische Rundschau 62, 11, 54-59.

Haase, A.; Herfert, G.; Kabisch, S.; Steinführer, A. (2010): Reurbanisierung in ostdeutschen Grossstädten. Regionale, städtische und Quartiersanalysen unter besonderer Berücksichtigung demographischer Prozesse. In: disP The Planning Review 46, 180, 24-35. https://doi.org/10. $1080 / 02513625.2010 .10557061$

Held, T.; Mäding, A. (2020): Das Ende der Reurbanisierung? Aktuelle Trends auf dem Wohnungsmarkt der Stadtregion Stuttgart. In: Stadtforschung und Statistik 33, 1, 29-36.

Herfert, G.; Osterhage, F. (2012): Wohnen in der Stadt: Gibt es eine Trendwende zur Reurbanisierung? Ein quantitativ-analytischer Ansatz. In: Brake, K.; Herfert, G. (Hrsg.): Reurbanisierung. Materialität und Diskurs in Deutschland. Wiesbaden, 86-112. 10.1007/ 978-3-531-94211-7_6

Hesse, M. (2010): Reurbanisierung oder Metropolisierung? Entwicklungspfade, Kontexte, Interpretationsmuster zum aktuellen Wandel der Grossstadtregionen. In: disP - The Planning Review 46, 180, 36-46. https://doi.org/10. 1080/02513625.2010.10557062

Hesse, M.; Scheiner, J. (2009): Residential Location, Mobility and the City: Mediating and Reproducing Social Inequity. In: Ohnmacht, T.; Maksim, H.; Bergman, M. M. (Hrsg.): Mobilities and Inequality. London, 187-206. https://doi.org/10.4324/9781315595719

Hirschle, M.; Schürt, A. (2008): Suburbanisierung ... und kein Ende in Sicht? Intraregionale Wanderungen und Wohnungsmärkte. In: Informationen zur Raumentwicklung 3/4, 211-227.

Holz-Rau, C.; Scheiner, J. (2015): Mobilitätsbiografien und Mobilitätssozialisation: Neue Zugänge zu einem alten Thema. In: Scheiner, J.; Holz-Rau, C. (Hrsg.): Räumliche Mobilität und Lebenslauf. Studien zu Mobilitätsbiografien und Mobilitätssozialisation. Wiesbaden, 3-22. https:// doi.org/10.1007/978-3-658-07546-0_1

Holz-Rau, C.; Scheiner, J.; Sicks, K. (2014): Travel Distances in Daily Travel and Long-Distance Travel: What Role is Played by Urban Form? In: Environment and Planning A 46, 2, 488-507. https://doi.org/10.1068/a4640

Hopkins, D.; Stephenson, J. (2014): Generation Y mobilities through the lens of energy cultures: a preliminary exploration of mobility cultures. In: Journal of Transport Geography 38, 88-91. https://doi.org/10.1016/j.jtrangeo. 2014.05.013

Hubbard, P. (2009): Geographies of studentification and purpose-built student accommodation: Leading sepa- 
rate lives? In: Environment and Planning A 41, 8, 1903-1923. https://doi.org/10.1068/a4149

Hurrelmann, K. (2012): Sozialisation. Das Modell der produktiven Realitätsverarbeitung. Weinheim.

Kabisch, S.; Steinführer, A.; Haase, A. (2012): Reurbanisierung aus soziodemographischer Perspektive: Haushalte und Quartierswandel in der inneren Stadt. In: Brake, K.; Herfert, G. (Hrsg.): Reurbanisierung. Materialität und Diskurs in Deutschland. Wiesbaden, 113-129. https:// doi.org/10.1007/978-3-531-94211-7_7

Karsten, L. (2003): Family Gentrifiers: Challenging the City as a Place Simultaneously to Build a Career and to Raise Children. In: Urban Studies 40, 12, 2573-2584. https:// doi.org/10.1080/0042098032000136228

Köppen, B. (2008): Reurbanisierung als Hoffnung der Städte im demographischen Wandel? In: Maretzke, S. (Hrsg.): Städte im demografischen Wandel: wesentliche Strukturen und Trends des demografischen Wandels in den Städten Deutschlands. Wiesbaden, 31-40. = Materialien zur Bevölkerungswissenschaft 125 .

Kholodilin, K. A. (2017): Wanderungssalden der deutschen Metropolen. In: Der Landkreis 87, 1-2, 45-48.

Kramer, C. (2019): Studierende im städtischen Quartier - zeit-räumliche Wirkungen von temporären Bewohnern und Bewohnerinnen. In: Henckel, D.; Kramer, C. (Hrsg.): Zeitgerechte Stadt: Konzepte und Perspektiven für die Planungspraxis. Hannover, 281-310. = Forschungsberichte der ARL 9.

Kramer, C.; Pfaffenbach, C. (2016): Should I Stay or Should I Go? Housing Preferences upon Retirement in Germany. In: Journal of Housing and the Built Environment 31, 2, 239-256. https://doi.org/10.1007/s10901-015-9454-5

Kühl, J. (2014): Faktoren der Wohnstandortwahl: Differenzierung von Wohnstandortanforderungen unterschiedlicher Nachfragegruppen. In: Danielzyk, R.; Lentz, S.; Wiegandt, C.-C. (Hrsg.): Suchst du noch oder wohnst du schon? Wohnen in polyzentrischen Stadtregionen. Münster, 25-44. = Schriften des Arbeitskreises Stadtzukünfte der Deutschen Gesellschaft für Geographie 12.

Matthes, G. (2016): Reurbanisierung und Verkehr. Münster. = Harburger Berichte zur Verkehrsplanung und Logistik 17. https://doi.org/10.15480/882.1365

Milbert, A. (2017): Wie viel (Re-)Urbanisierung durchzieht das Land? Bonn. = BBSR-Analysen KOMPAKT 07/2017.

Milbert, A.; Sturm, G. (2016): Binnenwanderungen in Deutschland zwischen 1975 und 2013. In: Informationen zur Raumentwicklung 2, 121-144.

Moos, M. (2016): From gentrification to youthification? The increasing importance of young age in delineating highdensity living. In: Urban Studies 53, 14, 2903-2920. https://doi.org/10.1177/0042098015603292
Münter, A.; Osterhage, F. (2017): Der regionale Wohnungsmarkt der Stadt Aachen: grenzenlos oder begrenzt? Dortmund. = ILS-Trends 1/2017.

Mulder, C. H.; Hooimeijer, P. (1999): Residential Relocations in the Life Course. In: van Wissen, L. J. G.; Dykstra, P. A. (Hrsg.): Population Issues. An Interdisciplinary Focus. New York, 159-186. https://doi.org/10. 1007/978-94-011-4389-9_6

Myers, S. M. (1999): Residential Mobility as a Way of Life: Evidence of Intergenerational Similarities. In: Journal of Marriage and Family 61, 4, 871-880. https://doi.org/10. 2307/354009

Ochs, B. (2016): Jetzt geht es wieder raus aufs Land. In: Frankfurter Allgemeine Zeitung Online. https://www. faz.net/aktuell/wirtschaft/wohnen/deutsche-landgebieteerhalten-wieder-zuwachs-14430840.html (23.06.2021).

Osterhage, F. (2018): The End of Reurbanisation? Phases of Concentration and Deconcentration in Migratory Movements in North Rhine-Westphalia. In: Comparative Population Studies 43, 131-156. https://doi.org/10.12765/ CPoS-2018-10

Sander, N. (2014): Internal Migration in Germany, 19952010: New Insights into East-West Migration and Reurbanisation. In: Comparative Population Studies 39, 2 , 217-246. https://doi.org/10.12765/CPoS-2014-04

Scheiner, J.; Sicks, K.; Holz-Rau, C. (2014): Generationsübergreifende Mobilitätsbiografien - Dokumentation der Datengrundlage. Eine Befragung unter Studierenden, ihren Eltern und Großeltern. Dortmund. = Raum und Mobilität - Arbeitspapiere des Fachgebiets Verkehrswesen und Verkehrsplanung 29.

Schendera, C. (2008): Regressionsanalyse mit SPSS. München. https://doi.org/10.1524/9783486710625

Scholich, D. (2019): Reurbanisierung zwischen Wunsch und Wirklichkeit. Ein Blick auf nordwestdeutsche Städte und Regionen - Eine Einführung. In: Scholich, D. (Hrsg.): Reurbanisierung zwischen Wunsch und Wirklichkeit. Ein Blick auf nordwestdeutsche Städte und Regionen. Hannover, 5-26. = Arbeitsberichte der ARL 27.

Siebel, W. (2010): Bedingungen der Reurbanisierung. In: disP - The Planning Review 46, 180, 106-114. https:// doi.org/10.1080/02513625.2010.10557067

Siedentop, S. (2018): Reurbanisierung. In: Rink, D.; Haase, A. (Hrsg.): Handbuch Stadtkonzepte. Analysen, Diagnosen, Kritiken und Visionen. Opladen, 381-403.

Siedentop, S.; Zakrzewski, P.; Stroms, P. (2018): A childless urban renaissance? Age-selective patterns of population change in North American and German Metropolitan areas. In: Regional Studies, Regional Science 5, 1, 1-20. https://doi.org/10.1080/21681376.2017.1412270 
Simons, H.; Weiden, L. (2016): Schwarmverhalten, Reurbanisierung und Suburbanisierung. In: Informationen zur Raumentwicklung 3, 263-273.

Smith, D. (2005): 'Studentification': the gentrification factory? In: Atkinson, R.; Bridge, G. (Hrsg.): Gentrification in a global context: the new urban colonialism. London, 72-89.

Stadt Dortmund, Amt für Wohnen (Hrsg.) (2020): Wohnen in Dortmund und der Region - Eine Wanderungsmotivuntersuchung. Dortmund.

Stadt Wolfsburg (2020): Wanderungsmotivbefragung 2018. Ergebnisse der Befragung von Zuzügen nach und Wegzügen aus Wolfsburg. Wolfsburg.

Thomas, E.; Serwicka, I.; Swinney, P. (2015): Urban demographics. Why people live where they do. London.

von Einem, E. (2016): Explodierende Wohnwünsche: Befragung der nächsten Generation. In: von Einem, E. (Hrsg.): Wohnen: Markt in Schieflage - Politik in Not. Wiesbaden, 145-158. https://doi.org/10.1007/978-3658-11757-3_7

Weller, G. (2016): Wohin zieht es Karlsruher Studierende nach ihrem Abschluss? Zukunftspläne und Wohnwünsche. Karlsruhe. Zulassungsarbeit zum Ersten Staatsexamen im Fach Geographie am Karlsruher Institut für Technologie (unveröffentlicht). 